\title{
Vier Jahre HeilberufeSCIENCE
}

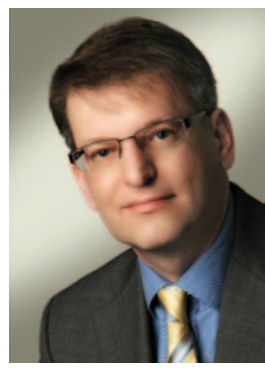

Seit mittlerweile 4 Jahren erscheint HeilberufeSCIENCE regelmäßig alle 3 Monate. Die regulären Ausgaben werden zusätzlich durch zwei Kongress-Sonderhefte mit den Beiträgen des Kongresses „Pflege“ in Berlin und des Gesundheitspflegekongress in Hamburg ergänzt. Im Laufe dieser 4 Jahre hat sich HeilberufeSCIENCE vom anfänglichen Supplement zu „Heilberufe - Das Pflegemagazin“ zum eigenständigen wissenschaftlichen Online-Journal mit verblindetem peer-review-Verfahren entwickelt. Dies spiegelt sich auch in den zunehmenden Listungen wieder, so hat z. B. CINAHL die Indizierung ab Ende 2012 zugesagt. Damit verbunden stieg auch kontinuierlich die Zahl der eingereichten Manuskripte mit hochwertigen Beiträgen.

Deswegen können wir Ihnen in dieser Ausgabe vier Originalarbeiten, zwei Übersichtsarbeiten und zwei wissenschaftliche Kurzmitteilungen bieten. Darunter befindet sich auch wieder ein englischsprachiger Beitrag von Hazelwood et al., die sich in einer systematischen Übersichtsarbeit mit den Interventionen auseinander setzen, die Familienmitglieder in das Management von krebs- und therapieinduzierten Symptomen bei der Behandlung von Tumorpatienten einbinden. Zuvor präsentieren Roth et al. in einer Originalarbeit die Ergebnisse der Analyse der Homepages aller sächsischen Akutkrankenhäuser hinsichtlich der Nutzung der Homepage zu Marketingzwecken. In einer weiteren Originalarbeit wurde von Melanie Messer untersucht, wie pflegende Angehörige die Inkontinenz ihres an AlzheimerDemenz erkrankten Ehepartners erleben. Kern et al. gehen darauf ein, wie Mitarbeiter in der Pflege Veränderungsprozesse bei der Umstrukturierung einer Einrichtung von stationärer hin zu ambulanter Versorgung wahrnehmen und welche Rolle dabei die Kommunikation mit den Mitarbeitern spielt. Reidl et al. setzen sich in einer Übersichtsarbeit mit der Berücksichtigung gerontologischer Aspekte in der Tourismusforschung auseinander und zeigen umfassenden weiteren Forschungsbedarf auf diesem Gebiet auf. Prein \& Mann stellen die Ergebnisse einer Befragung an über 800 Privatpersonen zum Informationsbedarf und -verhalten der Zielgruppe 50plus bei Pflege- und Gesundheitsthemen dar.

In wissenschaftliche Kurzmitteilungen gehen Lindeman et al. auf die Ergebnisse einer Befragung zu körperlichsportlichen Aktivitäten von Physiotherapieschülern ein und Sawetzki et al. präsentieren die Ergebnisse einer Analyse der Sturzereignisse in einem Krankenhaus der kardiologischen und herzchirurgischen Maximalversorgung.

An dieser Stelle möchte ich Sie wieder einladen, diese acht Beiträge dieser Ausgabe von HeilberufeSCIENCE kritisch zu lesen. Nutzen Sie auch die Gelegenheit, einen Blick in das Anfang November erschienene HeilberufeSCIENCESonderheft mit den Abstracts der Poster, wissenschaftlichen Vorträge und Fachbeiträge des 10. Gesundheitspflegekongress zu werfen.

Zum Abschluss wünsche ich Ihnen eine schöne Weihnachtszeit und alles Gute für das Jahr 2013!

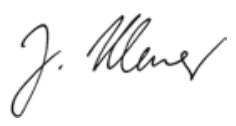

